\title{
Review of two books on salutogenesis
}

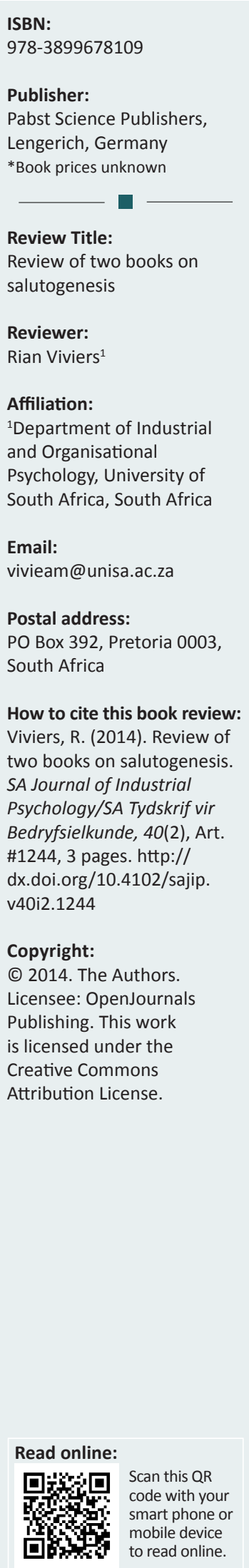

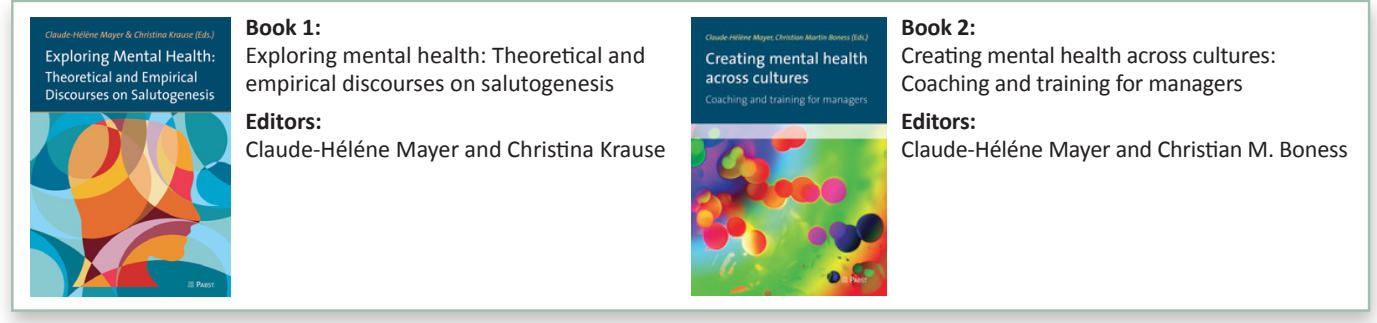

\section{Introduction}

The shift from a pathogenic to a salutogenic approach towards health and well-being started in the late 1970s with the work of Antonovsky (1979, 1985, 1987a, 1987b, 1990, 1993) when he introduced the salutogenic paradigm that focuses on health rather than disease. During these years, he conceptualised the construct of sense of coherence to reflect a person's orientation towards handling multiple stressors.

Ground-breaking work on the salutogenic construct has also been done in South Africa by Strümpfer (1990, 1995, 2006) and others (Wissing \& Van Eeden, 1997). Since the 1980s, many international as well as local research projects have been undertaken by researchers and scholars on the various salutogenic constructs (see Coetzee \& Viviers, 2007; Viviers \& Coetzee, 2007).

Throughout the past three decades, the construct of sense of coherence remained alive. Continued research on sense of coherence is evident not only in current literature, but it is also evident in conference proceedings. The recent publication of the two books under review is further evidence that the construct of sense of coherence is alive and well and still relevant in the world of work in 2014. Whilst the first book is a theoretical exploration of mental health with the focus on discourses on salutogenesis, the second book focuses on the creation of mental health with the main aim to train and coach managers across different cultures in a sense of coherence.

\section{General overview: Book 1}

The first book, Exploring mental health: Theoretical and empirical discourses on salutogenesis, (Mayer \& Krause 2012) is a compilation of nine articles covering a variety of disciplines in the exploration of the construct. In their editorial, the editors provide the reader with a detailed orientation to health and its paradigms, and they also touch on concepts of health in the broader positive-psychology context. The remainder of the editorial section is dedicated to a thorough explanation of salutogenesis and a sense of coherence.

The first article by Mayer deals with sense of coherence and the ability of managers to handle conflict in organisations. Empirical findings based on quantitative and qualitative data show how managers in South Africa deal with conflict management while trying to maintain health and well-being in the workplace.

The article by Krause and Fidelman aims to promote mental health in young children through the use of the salutogenic approach. They offer practical steps that teachers can utilise to boost the well-being of children and to strengthen children's' self-worth from an early age onwards.

The third article by Feldman, Davidson and Margalit addresses the mental health of college students and offers insights into the promotion of hope, sense of coherence and self-efficacy amongst this specific target group. The salutogenic paradigm within the college environment as setting is discussed in this article, followed by a systematic discussion of the different stages of the Hope workshop offered by the facilitators. 
Hüther and Flaig shift the focus to self-healing and provide a neurobiological perspective on healing, promoting the idea that all healing is essentially self-healing. The reader is taken through aspects such as the dilemma of obstructing selfhealing processes, the reactivation of these powers, and also the implementation of self-healing practices.

The next article (Hintermair \& Wälder) deals with a sense of coherence, emotional stress and the coping strategies of the hard of hearing. The empirical study reaffirms the role of sense of coherence in creating a lifestyle that is health orientated - even amongst people with hearing problems.

Buch introduces shamanism as applied salutogenesis practice and investigates whether shamanic practices inherently use salutogenic principles. Through her extensive literature study, she compares selected elements of shamanism with those principles as captured in the salutogenic literature by Antonovsky.

Bahrs offers an interesting perspective on prescription drugs in his article, which follows a medical-sociological examination of the usage and effectiveness of prescription drugs. In his results, he reports insightful findings based on the transcription of videotapes and consultations with doctors and patients.

The salutogenic perspective on mental health is extended across the life span by Eriksson, Sagy and Lindström. In their article, they also explore and deal with the cultural aspects related to the construct of sense of coherence. The empirical study utilises the results of published research paper over a period of 12 years as well as additional papers up to 2010 to address and answer questions they posed on salutogenic theory and the construct of sense of coherence.

The last contribution to the book is a chapter by Petzold which addresses the basics of systemic coherence regulation. The article also provides a discourse on dynamic and systemic approaches to salutogenesis. The author proposes a model of communicative coherence regulation to address the issue of how human beings can progress towards health.

\section{General overview: Book 2}

The second book, Creating mental health across cultures: Coaching and training for managers (Mayer \& Krause 2013), is a practical workbook. The book consists of six chapters. Chapter one serves as an introduction to health and wellbeing and explains the aims and content of the book. It provides the METHO (Mental Health in Transcultural Organisations) coaching and training model that forms the basis of the book. The focus stays mainly on Antonovsky's construct of sense of coherence.

Chapter two forms a broad introduction to the world of health and well-being with the focus on developing and promoting these aspects. In this chapter, salutogenesis and a sense of coherence forms the basis of the text, and various sections are devoted to the promotion of a sense of coherence.
In chapter three, transcultural competence and identities are introduced with the emphasis on managers taking a up professionally leading roles even across cultures. Managerial values and identities as well as managerial spirituality and religion also receive attention in this chapter.

Chapter four tackles the development of transcultural conflict-management competencies. The chapter not only explores management of conflict across cultures, but draws implications for managing conflict while staying healthy mentally. A section of the chapter is allocated to conflict and identities within organisations. There is also a section on light and shadow in organisations, addressed from a Jungian perspective. The last section of this chapter is devoted to techniques to de-escalate communication in striving for better transcultural processes to handle conflict at work.

The fifth chapter promotes healthy organisational cultures by providing an overview of systemic organisational cultures and explaining and exploring systemic concepts within organisations. This chapter includes brief overviews of aspects such as a resource-focus for empowerment as well as perspectives on growth-orientation for organisations.

The sixth and last, one-page chapter serves as a conclusion and provides reference to perspectives on a healthy, transcultural management future.

\section{Overall impression and conclusion}

As employee wellness and mental health of employees play an important role in the effective functioning of organisations, organisations are encouraging support for and the development of the general physical as well as mental health of employees. Many organisations do not only support physical training programmes, but they also engage their employees in workshops to enable them to maintain a healthy work-life balance in order to stay physically and mentally healthy, to optimise their personal physical and mental health and thus to function as a fully optimal and productive workforce.

The purpose of book one is to provide a theoretical overview of salutogenesis and a sense of coherence with explorations of sense of coherence in selected research areas and across disciplines. Although this might sound too academic for the practitioner, trainer or teacher in organisational context, the contents is of such a nature that theory and concepts are conveyed to the reader in a straightforward and clear manner. Anyone with an interest in the field of health psychology will surely relate to the content and find the diverse articles thought provoking. The book comprises a number of articles reflecting theoretical and empirical work, and scholars of psychology and academics should find the content relevant. Managers within organisations would equally be able to relate to the contents as it provides a thorough, scientific basis for personal self-development initiatives in the health paradigm. In fact, it forms the basis and foundation for engaging in the practical work as offered in book two. 
Book two offers the trainer, coach or individual person the opportunity to engage on a practical level with theories and constructs of mental health. The focus of the book is on salutogenesis and a sense of coherence. In all the chapters, the reader is introduced to a brief, easily comprehensible theoretical section that provides an overview of the topic under discussion. The practical exercises require introspection and reflection on the side of participants, and they are systematically guided through numerous exercises in each chapter to contemplate their own current perspectives, views, styles and manners of functioning. Exercises are aimed at eventually narrowing the focus in enhancing an in-depth understanding of self in relationship to others and own issues. This process takes the individual on a journey to explore the development of mental health and to engage in more healthy managerial and personal practices on a daily basis.

The two books form a cohesive package that would benefit students in positive psychology, lecturers offering curricula in health psychology, as well as industrial and organisational psychologists and practitioners in the organisational contexts. The books would provide these readers with a better understanding of salutogenesis and a sense of coherence as elements of the broader health paradigm. The workbook offers a valuable practical aid in integrating theoretical salutogenic concepts into managerial practices on a daily basis.

Critique against these two volumes is that the focus is solely on one salutogenic construct as conceptualised by Antonovsky $(1985,1987,1990)$. Work done by Strümpfer $(1990,1995)$ and Wissing and Van Eeden (1997) in broadening the salutogenic paradigm is only mentioned in the opening chapters of book one. Additional salutogenic constructs worth exploring, such as hardiness, locus of control and self-efficacy, are merely mentioned. It is a pity that these well-researched constructs have been ignored, especially in book two, as numerous individuals might have benefitted from capitalising on different and diverse health theories and approaches.

\section{Acknowledgements}

I would like to acknowledge the authors of the two academic books who provided me with interesting reading for the book review.

\section{Competing interests}

The author declares that he has no financial or personal relationship(s) that may have inappropriately influenced him in writing this article.

\section{References}

Antonovsky, A. (1979). Health, stress and coping. San Francisco: Jossey-Bass.

Antonovsky, A. (1985). The life circle, mental health and the sense of coherence. Israel Journal of Psychiatry and Related Sciences, 22, 273-280.

Antonovsky, A. (1987a). The salutogenic perspective: Toward a new view of health and illness. Advances: The Journal of Mind-Body Health, 4, 47-55.

Antonovsky, A. (1987b). Unraveling the mystery of health. San Francisco: Jossey-Bass.

Antonovsky, A. (1990). A somewhat personal odyssey in studying the stress process. Stress medicine, 6, 71-80. http://dx.doi.org/10.1002/smi.2460060203

Antonovsky, A. (1993). Complexity, conflict, chaos, coherence, coercion and civility. Social Science Medicine, 37, 969-981. http://dx.doi.org/10.1016/02779536(93)90427-6

Coetzee, S. \& Viviers, R. (2007). An overview of research on positive psychology in South Africa. South African Journal of Psychology, 37(3), 470-490. http://dx.doi. org/10.1177/008124630703700307

Mayer, C-H., \& Krause, C. (Eds.) (2012). Exploring mental health: Theoretical and empirical discourses on salutogenesis. Lengerich: Pabst Science Publishers.

Mayer, C-H., \& Krause, C.M. (Eds.). (2013). Creating mental health across cultures: Coaching and training for managers. Lengerich: Pabst Science Publishers.

Strümpfer, D.J.W. (1990). Salutogenesis: A new paradigm. South African Journal of Psychology, 20, 45-52. http://dx.doi.org/10.1177/008124639002000406

Strümpfer, D.J.W. (1995). The origins of health and strength: From 'salutogenesis' to 'fortigenesis'. South African Journal of Psychology, 25, 81-89. http://dx.doi. org/10.1177/008124639502500203

Strümpfer, D.J.W. (2006). Positive emotions, positive emotionality and their contribution to fortigenic living: A review. South African Journal of Psychology, 36(1), 144-167. http://dx.doi.org/10.1177/008124630603600109

Viviers, A.M. \& Coetzee, S. (2007). South African universities, research and positive psychology. South African Journal of Higher Education, 21(4), 764-780.

Wissing, M.P. \& Van Eeden, C. (September, 1997). Psychological well-being: A fortigenic conceptualization and empirical clarification. Paper presented at the 3rd Annual Congress of the Psychological Society of South Africa, Durban, South Africa. 